\title{
Aeromonas hydrophila septicaemia and muscle abscesses associated with immunosuppression
}

\author{
N.J. Slevin, ${ }^{1}$ B.A. Oppenheim ${ }^{2}$ and D.P. Deakin ${ }^{1}$
}

Departments of ${ }^{1}$ Radiotherapy and ${ }^{2}$ Microbiology, Christie Hospital and Holt Radium Institute, Wilmslow Road, Withington, Manchester M20 9BX, UK.

\begin{abstract}
Summary: We report a case of septicaemia and muscle abscesses due to Aeromonas hydrophila. The patient was immunosuppressed due to Hodgkin's disease and treatment with cytotoxic chemotherapy. The abscesses resolved after prolonged antibiotic therapy. Organisms that were once considered rarely pathogenic are emerging as increasingly important in causing potentially lethal infections in immunosuppressed patients.
\end{abstract}

\section{Introduction}

Aeromonas hydrophila is a Gram-negative bacillus commonly found in soil and water; it is responsible for a number of diseases in cold-blooded animals such as 'red leg' disease in frogs and ascites in carp. ${ }^{1}$ In humans it is associated with an acute diarrhoeal illness which is self-limiting. ${ }^{2}$ The organism has been isolated in $4.2 \%$ of 1004 unselected faecal specimens from patients with gastrointestinal symptoms or their asymptomatic contacts. ${ }^{3}$ Systemic infection with Aeromonas hydrophila is uncommon but has been reported in immunosuppressed patients with malignant ${ }^{4}$ and hepatobiliary disease. ${ }^{5}$ In some cases it may be associated with ecthyma gangrenosum, the necrotizing skin lesion usually caused by infection with Pseudomonas aeruginosa. ${ }^{6}$

The typical presentation of pyogenic muscle abscess is with a painful swelling in the buttock or thigh, which is woody hard, evolving to become fluctuant. ${ }^{7}$ In both temperate and tropical countries the organism responsible for pyogenic muscle abscesses is predominantly Staphylococcus aureus. ${ }^{8}$

We report a case of septicaemia and multiple muscle abscesses due to Aeromonas hydrophila in a patient having cytotoxic chemotherapy for Hodgkin's disease.

\section{Case report}

A 62 year old man was admitted with a 5-day history of rigors, sore throat and painful leg

Correspondence: N.J. Slevin, M.R.C.P., F.R.C.R. Accepted: 20 April 1988 muscles. He had commenced combination oral chemotherapy for recurrent Hodgkin's disease 11 days before admission. He looked unwell, was pyrexial $\left(39^{\circ} \mathrm{C}\right)$ and had florid oral candidiasis. The muscles of his thighs and calves were extremely tender, but muscle power was preserved. The white blood cell count was $0.2 \times 10^{9} / 1$, and a clinica diagnosis of septicaemia was made. $\mathrm{He}$ was. commenced on intravenous ceftazidime and bloo cultures subsequently grew Aeromonas hydrophila, sensitive to this antibiotic. Serum muscle enzyme estimation showed a creatine kinase level of $139 \mathrm{IU} / \mathrm{l}$ (normal 20-130) and a normal aspartate transaminase level. Over the following 2 weeks his general condition improved, the temperature settled after 5 days, the muscles became less tender and white cell count rose to $3.2 \times 10^{9} / 1$. The antibiotic was stopped after 14 days.

On day 14 of his admission he complained of a painful lump in his left thigh. On examination there was a $10 \times 5 \mathrm{~cm}$ hard tender mass in the left quadriceps muscle. Aspiration was attempted but was unsuccessful. Over the next 4 days he developed further hard tender lumps in the right thigh and left calf muscles and he became pyrexial $\left(40^{\circ} \mathrm{C}\right)$. Blood cultures were taken and on day 18 he was commenced on intravenous cefuroxime. On this occasion blood and faecal cultures were normal. As his clinical condition did not improve over several days intravenous netilmicin was introduced. On day 28 the mass in the left thigh was noted to be fluctuant and $15 \mathrm{ml}$ of pus was aspirated; the pus was subsequently shown to be sterile. Over the next 2 weeks his muscle abscesses resolved, his mobilization gradually improved and he remained apyrexial. On follow-up, 2 months after discharge,

(C) The Fellowship of Postgraduate Medicine, 1988 
there was no evidence of recrudescence of the muscle abscesses.

\section{Microbiology}

Blood cultures were performed using the BACTEC radiometric method. An oxidase positive Gramnegative rod was isolated from both aerobic and anaerobic bottles and this was identified as Aeromonas hydrophila using the API 20E system. Antibiotic susceptibility testing by the Stokes method $^{9}$ showed the organism to be sensitive to gentamicin and netilmicin, cefuroxime, ceftazidime and ciprofloxacin, and resistant to ampicillin and piperacillin.

\section{Discussion}

Pyogenic muscle abscess is rare in non-tropical countries. Muscle abscess due to Aeromonas hydrophila has been described in only four previous cases. ${ }^{10}$ In three of the four cases the cause of infection was confirmed at post-mortem. The fourth case is the only other reported survivor from

\section{References}

1. Parker, M.T. Septic infections due to Gram-negative aerobic bacilli. In: Topley and Wilson's: Principles of Bacteriology, Virology and Immunity. Edward Arnold, London, 1983, 3: pp. 302-303.

2. Agger, W.A., McCormick, J.D. \& Gurwith, M.J. Clinical and microbiological features of Aeromonas hydrophila - associated diarrhea. J Clin Microbiol 1985, 21: 909-913.

3. Millership, S.E., Curnow, S.R. \& Chattopadhyay, B. Faecal carriage rate of Aeromonas hydrophila. J Clin Pathol 1983, 36: 920-923.

4. Harris, R.L., Fainstein, V., Elting, L., Hopfer, R.L. \& Bodey, G.P. Bacteremia caused by Aeromonas hydrophila in hospitalised cancer patients. Rev Infect Dis 1985, 7: 314-320.

5. Colaco, C. Aeromonas hydrophila liver abscess. Lancet 1982, i: 680 (letter).

6. Ketover, B.P., Young, L.S. \& Armstrong, D. Septicemia due to Aeromonas hydrophila. Clinical and immunological aspects. J Infect Dis 1973, 127: 284-290.
Aeromonas hydrophila muscle abscesses; the leg muscle abscesses were drained by bilateral fasciotomies and the patient recovered after prolonged antibiotic treatment with gentamicin and trimethoprim-sulphamethoxazole.

This case illustrates the classic features of muscle abscess, with a tender indurative mass in the thigh accompanied by fever. Despite extensive myofibril destruction marked elevation of skeletal muscle enzymes is uncommon as is apparent in this case.

This patient was immunosuppressed with a granulocytopaenia induced by cytotoxic chemotherapy. Organisms that were once considered rarely pathogenic are emerging as increasingly important in causing lethal infections in immunosuppressed patients. If muscle abscess is diagnosed in an immunosuppressed patient, infection with Aeromonas hydrophila should be considered. In spite of in vitro sensitivity to cefuroxime in this case, the fever did not settle until netilmicin was introduced. The majority of isolates of Aeromonas are sensitive to aminoglycosides. ${ }^{4,6,11}$ If the diagnosis of Aeromonas hydrophila muscle abscess is made a prolonged clinical course (6 weeks in this case, 6 weeks post drainage in the other reported survivor) should be anticipated.

7. Watts, R.A., Hoffbrand, B.I., Paton, D.F. \& Davis J.C. Pyomyositis associated with human immune deficiency virus infection. $\mathrm{Br} \mathrm{Med} J$ 1987, 294: 1524-1525.

8. Gibson, R.K., Rosenthal, S.J. \& Lukert, B.P. Pyomyositis - increasing recognition in temperate climates. Am J Med 1984, 77: 768-772.

9. Stokes, E.J. \& Waterworth, P.M. Association of Clinical Pathologists. Broadsheet No. 55 (revised 1972).

10. Kratzke, R.A. \& Golenbock, D.T. Pyomyositis and hepatic abscess in association with Aeromonas hydrophila sepsis. Am J Med 1987, 83: 347-349.

11. Von Graevenitz A. \& Mensch, A. The genus Aeromonas in human bacteriology: report of 30 cases and review of the literature. $N$ Engl $J$ Med 1968, 278: 245-249. 